\title{
Opioid and Hedgehog signalling pathways converge to modulate OA
}

As one of the four main opioid receptor subtypes, $\kappa$-opioid receptor (KOR) is involved in pain processing, consciousness and addiction. However, unlike other opioid receptors, KOR is also expressed by pre-chondrocytes and seems to be involved in preventing cartilage degeneration and osteoarthritis (OA).

"Our previous studies laid the foundation for establishing KOR signalling as a potential therapeutic target for not only pain relief, but also for prevention of joint cartilage degeneration. However, the precise molecular mechanisms involved in KOR regulation of joint development and health remained largely unsolved," explains Denis Evseenko, corresponding author of a new study published in Arthritis \& Rheumatology. "Our group was interested in finding a novel signalling mechanism of KOR modulation, as opioid receptor signalling could have therapeutic potential in treating degenerative joint disease."

$\mathrm{KOR}$ is a $\mathrm{G}$ protein-coupled receptor that can signal through secondary messengers that are known to inhibit the Hedgehog signalling pathway, which also has a role in cartilage degeneration and OA development. Evseenko and colleagues used the selective KOR agonist JT09, which is analgesic but has reduced addictive qualities, to determine if KOR signalling pathways affected Hedgehog signalling pathways.

In vitro, JT09 inhibited Hedgehog signalling in healthy and osteoarthritic human chondrocytes. In rats with partial medial meniscectomy-induced OA, intra-articular injection of JT09 reduced cartilage loss at 6 weeks compared with injection of saline and prevented degenerative changes to

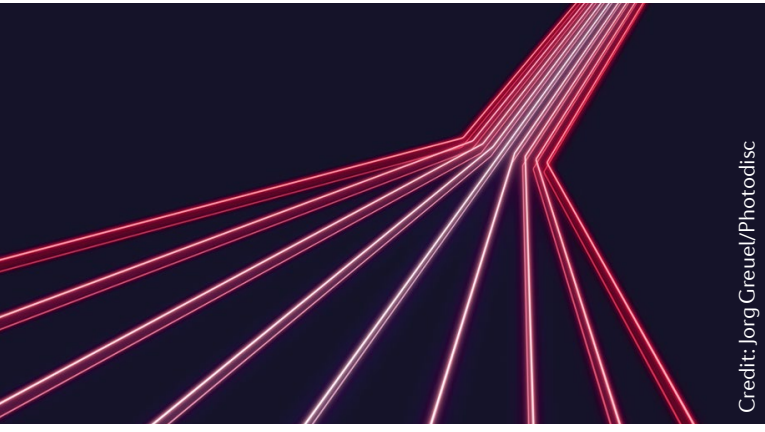

the structure of the articular surface. These changes were accompanied by a reduction in components of the Hedgehog signalling pathway in the articular cartilage.

"Our study defines a novel molecular mechanism for the role of the KOR in articular cartilage homeostasis and disease, providing a unifying mechanistic basis for the overlap in processes and pathologies that involve opioid and Hedgehog signalling," concludes first author Alexander Weber.

Joanna Clarke with injection of saline

5

ORIGINAL ARTICLE Weber, A. E. et al. Kappa pioids modulate hedgehog signaling to attenuate osteoarthritis. Arthritis Rheumatol. https://doi.org/10.1002/art.41250 (2020)

\section{Microbiota-derived metabolites help regulatory $B$ cells suppress arthritis}

Regulatory $B\left(B_{\text {reg }}\right)$ cells are important for maintaining immunological tolerance and for suppressing pathogenic responses in autoimmune diseases such as rheumatoid arthritis. New findings suggest that butyrate, a gut microbiota-derived short-chain fatty acid generated during fermentation of dietary fibres, can suppress arthritis by influencing the development and function of $B_{\text {reg }}$ cells.

"There have been many studies investigating how microbiota-derived metabolites affect $\mathrm{T}$ cell and myeloid

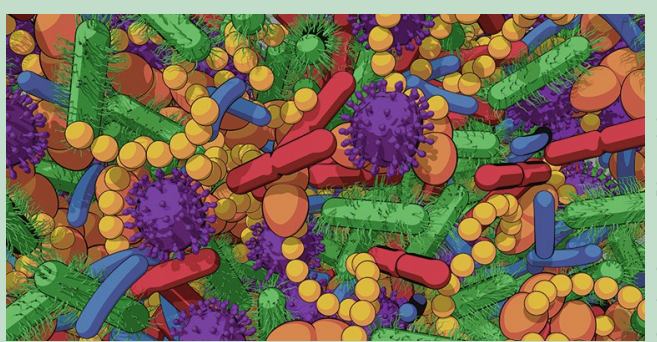

cell function but little work has been carried out to investigate how these metabolites modulate B cell function," explains Elizabeth Rosser, first author on the new study.

In a mouse model of experimental arthritis, the researchers found that dietary supplementation with butyrate could reduce arthritis severity in a manner dependent on IL-10-producing B cells. Indeed, butyrate supplementation skewed the B cell population in these mice towards a regulatory phenotype.

Using knockout mice, adoptive transfer experiments and chimeric mouse models, Rosser and colleagues pinpointed the involvement of the aryl-hydrocarbon receptor (AhR) in butyrate-mediated suppression of arthritis, including in the promotion of $B_{\text {reg }}$ cell function and suppression of germinal centre $B$ cell and plasmablast differentiation.
Further experiments showed that butyrate did not directly upregulate AhR activity in B cells but instead increased the levels of the metabolite 5-hydroxyindole-3-acetic acid (5-HIAA) in the gut, potentially through shifting the gut microbiota profile towards an increased abundance of particular bacteria genera. 5-HIAA itself directly increased the activity of AhR in B cells. Notably, treatment with 5-HIAA suppressed arthritis development in wildtype mice, but not in mice with a B-cell specific deletion of AhR or in mice treated with a tryptophan hydroxylase inhibitor known to reduce 5-HIAA synthesis.

"Our data suggest that a high fibre diet and/or butyrate-supplementation might be anti-inflammatory in the context of arthritis," says corresponding author Claudia Mauri. "Modulating the production of gut-derived metabolites with dietary changes, prebiotics or probiotics could be immune-suppressive in the context of arthritis."

\section{Jessica McHugh}

ORIGINAL ARTICLE Rosser, E. C. et al. Microbiotaderived metabolites suppress arthritis by amplifying aryl-hydrocarbon receptor activation in regulatory B cells. Cell Metab. 31, 837-851 (2020) 